\title{
Homoeopathic challenge addressed by some striking facts and research findings culminates in a generalized concept of medicines
}

\begin{abstract}
High dilution homoeopathy continues to challenge the prevalent concept of medicine for more than two centuries. It is really puzzling how even without original medicinal substances different homoeopathic preparations can differ from one another and cure diseases. The skeptics latch on to this absence and go on branding those cures as placebo effect. But, instead of denying inconvenient facts some open minded scientists are engaged in addressing the homoeopathic challenge, namely, answering two most fundamental questions:
\end{abstract}

1. How various super-diluted substances differ from one another?

2. What is the clue to their medicinal value vis-à-vis how can they cause cures?

This article shows that skeptics' objections are logically refutable. But, the real necessity is scientific explanation. Thanks to three striking properties of water the cloud seems to be thinning out. These properties are discussed in explaining homoeomystery. They answer the first question as: Various super-diluted substances may be chemically just water but they differ from one another in their molecular structures.

Another special property of water and template principle of biology lead to the structural model of medicines answering the second question as: A substance is to be recognized as a medicine if it has the capability of curing disease(s) while its medicinal property is to be attributed to molecular structure of vehicle like water or of distinct chemical substance, when it exists.

Finally, structural concept in non-homoeopathy, like locking principle of antibiotics and shift of focus from chemical formula to physical structure suggest structures as generalized form of medicine.

Keywords: challenge of homoeopathy, structural model, generalized concept of medicines
Volume 3 Issue 2 - 2016

Mahata CR

Hony Emeritus Scientist, IIEST Shibpur, India

Correspondence: Mahata CR, Hony Emeritus Scientist, IIEST Shibpur, India,Tel +9l 943373 9180, Email crmahata@gmail.com

Received: January 29, 2016 | Published: February 25, 2016

\section{Introduction}

Homoeopathy is impressive because of two main reasons. It offers cheapest and yet a superior system of medicine. Firstly, regarding cheapness, one $5 \mathrm{ml}$ vial of 30c potency containing about 860 globules of size no. 20 (most frequently used globules) costs Rs 10/-. So, a dose of 4 globules will cost slightly less than Rs 0.05 , that is, 20 doses for a Rupee or 1300 doses for a USD! Thus, it is the cheapest and most affordable system of medicine. Secondly, regarding superiority, I have seen cures from severe bruises and tooth extraction without bleeding by Arnica Mont, relief from arthritis by Calcarea Phos, cure of prolapsed uterus by Sepia, of severe burning pain in urethra with blood in urine by Cantharis, to give only a few examples. In all these cases only a few doses with negligible cost were enough and there were no side effects. Veteran homoeopaths can cite many more cases of incredible cures. Such cases indicate superiority of homoeopathy over other kinds of medicines. That is why I do not agree with the skeptics. Moreover, skeptics' view of 'placebo effect' or 'mental cure' is logically untenable for the following reasons.

1. Skeptics forget the fact that homoeopathy benefits not only persons with thinking minds, but also babies, animals and plants. Cures in all cases cannot be attributed to power of mind.
So, 'placebo effect' or mental cure is an illogical proposition.

2. Moreover, there is no reason for a patient to think that only homeopathic pills (without any medicinal value, as per the skeptics) will be curative and not arbitrary main stream pills. In fact, such an effect was expected to be much stronger for pills of mainstream medicines to destroy utility of all real medicines. For, qualification of doctors, glamour of testing gadgets, medicines etc. all go in favor of the main stream to influence the thinking of a patient. So, diagnosis, prescription etc. would have been irrelevant issues. Any pill would have done the job. Still, skeptics hold that cure followed by mainstream pill is medicinal, whereas innumerable cures caused by potentised substances for over two centuries are mental. This is clearly a bias against homoeopathy violating the spirit of impartiality of science.

3. Mind also, to which they attribute cures, does not have any quantifiable active ingredient. The mechanism of action of mind in curing a disease is also not given by the advocates of placebo effect. As such, the skeptics' proposition is having the same drawbacks for which they stand against homoeopathy. It is merely a dogmatic opposition to homoeopathy. 
4. Cures by power of mind are stray intrusions into our day-today life giving us a glimpse of some transcendental principle realized and applied by yogis and saints. By and large, ordinary people cannot invoke this principle. Their approach to life is predominantly materialistic. Hence, their cures must be medicinal.

5. Nobody, including the advocates of placebo hypothesis, depends on placebo for curing his/her own illness. It implies that nobody 'really' believes in placebo-cure. So, this proposal is just frivolous.

Such logical rebuttal of skeptics will give a shot in the arm of any homoeo-lover. But, the real necessity is a scientific explanation. Clinical successes for over two centuries have not been able to give homoeopathy its legitimate status. Clinical research in homoeopathy starts with a big question mark because any medicine of potency above $12 \mathrm{c}$ taken for the purpose till now cannot be guaranteed by any test to be real. Conventional chemistry has no answer to how they can be identified, that is, differentiated from one another or what is the secret of their medicinal value. Original medicinal substance being diluted away these are chemical-free substances. So, where is the medicinal value of the super-diluted substances? Lack of satisfactory scientific explanation for such fundamental questions continues to embarrass homoeopathy. Its social perception also is not as respectable as it should be. All the clinical successes achieved so far have not been able to change this situation. Evidence based statistical approach by passes the fundamental issues. As such it has not succeeded in producing the desired impact. Now, it is obvious that the situation cannot be altered without answering the fundamental questions.

\section{Answering the first question}

Initial difficulty: High dilution homoeopathy gives no scope to its researchers to hold on to any commonly understood medicinal substance, the starting material being diluted away. A simple calculation reveals ${ }^{1}$ that that one atom of Sulphur can be ascertained in a common medicine like Sulphur-30c when its mass exceeds the mass of about 15,132 Suns! And 30c is just a medium potency in homoeopathy. Given this mind-boggling figure research for scientific basis of homoeopathy appears to many persons like searching for a black cat in a dark room where no cat exists. Chemically speaking, any high potency medicine is nothing but the vehicle of potentisation (i.e. water). But, fact remains fact. They are also effective curative agents. Over and above, sometimes they give incredible results. A true scientist cannot brush aside inconvenient facts and cannot tell like a skeptic that they are all placebo/mental effect.

Various models have been advanced from time to time for explaining the mystery of homoeopathy. But we must not forget that a model for scientific basis of homoeopathy must be able to answer the two most fundamental questions:

1. How various super-diluted substances differ from one another?

2. What is the clue to their medicinal value vis-à-vis how can they cause cures?

Some models try to explain homoeopathy on the basis of light isotopes, presence of silica or dissolved gases. But, they do not give any clue for answering the second question, namely, how can they cause cures? So, they are kept out of consideration of this article.

Clue: Nevertheless a careful observation reveals that action of a potentised medicine is specific to the original medicine plus its potency. Natrum Mur 30c and Natrum Sulph 30c act differently on living bodies. Similarly, action of Silicea 6x differs from that of Silicea 200c. In fact, this gives a clue for unraveling the mystery of homoeopathy. It tells us that chemically all high potency medicines may be just the vehicle, still there is something which makes them different from one another. Then, the question is-What is that 'something'?

Arrival at that 'something': For arriving at that 'something' we need not resort to any wild guess. It is sufficient if water can hold some kind of impression/information about medicines in the form of medicine-plus-potency specific molecular structures. Such structures can make different medicines out of the same chemistry. 'Molecular Structure' (NOT chemical formula) will be that 'something' sought for. (How structures can serve as medicines is taken up later.) Now, the question is-can water hold impression/information about so many homoeopathic medicines along with their potencies? Yes. Water, the diluting vehicle, can do this job by virtue of the following three striking properties: (i) It contains reasonably stable structures at room temperature (ii) of very large numbers to hold impressions of very large number of homoeopathic medicines along with their potencies. (iii)inducible by various starting materials through various stages of serial dilution. (It is to be noted here that medicinal grade alcohol, which is also used as a vehicle of potentisation, contains about 10 to $15 \%$ water.) A detailed discussion of these three striking properties is given below.

a. Firstly, even from pretty old references ${ }^{2-5}$ we come to know that at room temperature water can have innumerable tiny floating ice crystals or icicles as stable structures at least up to $400 \mathrm{C}$. It is also corroborated by recent findings ${ }^{6}$ using various techniques like IR, UV-Vis, Fluorescence Microscopy, Light Scattering and Atomic Force Microscopy. Evidences of structures are also available from ref. ${ }^{7-9}$ Quantum Electro Dynamic (QED) coherent calculations by Giudice E $\operatorname{Del}^{9}$ (a quantum field theorist of condensed soft matter and called as Prometheus of the new science) and his co-authors ${ }^{10,11}$ as well as Yinnon C \& Yinnon $\mathrm{TT}^{12}$ show that, at room temperature, liquid water is a mixture of coherent (coherence domain, CD) and incoherent water. Germano $\mathrm{R}^{13}$ reports spontaneous organization of a certain fraction of the particles of water in spatially coherent domains (CDs), where they oscillate collectively in phase with a self-generated electromagnetic field, their density being same as that of ice. Further, coherent structures get stabilized by attraction between liquid molecules and recipient wall/ immersed object surface. Bano Ivan et al., ${ }^{14}$ also give a strong theoretical basis of CDs with the help of QED. This satisfies the first requirement of stable structures at room temperature.

b. Secondly, forms of these crystals are so varied in number that practically no two ice crystals are identical. ${ }^{3} \operatorname{Ref}^{15}$ also reports the same. However, as demonstrated by Elia V et al., ${ }^{6}$ low energy perturbations affect the coherent domains and this may cause variations in water crystals. This includes potentisation process of homoeopathy. This satisfies the second requirement of very large number of structures to hold impressions of very large number of homoeopathic medicines along with their potencies.

c. Lastly, the shape and size of these molecular clusters are influenced by impurities, ions of other substances and even large foreign molecules. ${ }^{3}$ A decisive amount of experimental data has now been collected concerning the physicochemical properties of water subjected to physical perturbations like Iteratively Nafionated Water (INW), ${ }^{16-18}$ Iteratively Filtered 
Water (IFW), ${ }^{18-21}$ effect of hydrophilic surfaces ${ }^{22,23}$ and Extremely Diluted Solutions (EDS). ${ }^{24-29} \operatorname{Ref}^{30-37}$ also indicate induced changes in structure of water. Further, V. Elia's group has 18 more publications which are enlisted as references at the end of ref. ${ }^{6}$ Very strikingly, transduction of DNA information occurs through water and electro-magnetic waves as reported by the Nobel Laureate Montagnier Luc et al., ${ }^{38}$ raising capability of water to a new height. Here also water has to have structural variations for DNA transduction in Polymerase Chain Reaction (PCR). This satisfies the third requirement of structures inducible by various starting materials through various stages of serial dilution.

Water structure hypothesis is also directly or indirectly supported by several other researchers. Barnard $\mathrm{GO}^{39}$ was possibly the first to hint at the structural concept though he named it as water polymer. But, his work lacked experimental support. Stupendously successful experimental results were reported by Boyd WE. ${ }^{40}$ But, explanation for the emanations he detected by his 'emanometer' were missing at that time. Much later, Chattopadhyay R et al., ${ }^{41}$ offered an explanation suggesting that it originated from structured vehicle getting excitation from ambient electromagnetic radiation. Anagnostatos GS et al., ${ }^{42}$ proposed his structural model calling the structured water (of vehicle of potentisation) as 'clathrates'. He described how clathrates are likely to be generated at different stages of serial dilution. Water, with a simple chemical formula of $\mathrm{H}_{2} \mathrm{O}$, has attracted the attention of many researchers for explaining its exceptional properties. It is evidenced by Chaplin $\mathrm{M}$ enlisting 2388 investigations on water. ${ }^{43}$ Attempts continue. Maity T et al., ${ }^{30,31}$ and Mahata $\mathrm{CR}^{32,33}$ showed experimentally that medicine-plus-potency specific water structures were present in medicines both below and above the Avogadro limit of 12c. Roy Rustum et al., ${ }^{34,35}$ firmly supports the structural concept with some experimental results. Davenas E et al., ${ }^{44}$ in their experiments with human basophile degranulation show that 'memory' of the original substance are retained in serial dilution followed by vortexing even beyond the Avogadro limit. It was not a work with medicines but it has relevance to the dilution/ potentisation of homoeopathy. They called it as 'water memory', but we like to call it induced structure. Similarly Rey Louis ${ }^{36}$ in his investigation with dilution of substance like $\mathrm{LiCl}$ and $\mathrm{NaCl}$ showed that the hydrogen-bonded network of water remained modified even in dilutions beyond Avogadro limit. We may assume that such modification will lead to variation in structure of molecular clusters of the vehicle. Montagnier L et al., ${ }^{38}$ demonstrated that even massively diluted (1012) DNA and RNA solutions continue to emit electromagnetic radiations like the un-diluted one. Ref ${ }^{41}$ offers a possible explanation of this phenomenon in terms of structures. Support to structural model for extremely diluted substances also comes from the work of Elia $\mathrm{V}$ et al. ${ }^{16}$ But, the structural model as a generalized concept was possibly first advanced by Mahata CR. ${ }^{45}$

These works suggest that it is possible to have different molecular structures with same chemical formula, that is, $\mathrm{H}_{2} \mathrm{O}$, the structures being specific to the original medicinal substance plus the degree of potentisation. So, the answer to the first question may be stated as: Various super-diluted substances may be chemically just water but they differ from one another in the molecular structure of water (the potentising vehicle).

It is worth mentioning here that only three major models are currently being investigated by scientists for explaining homoeomystery. ${ }^{46}$ First model - water clusters or clathrates; second model - coherent domains (CD) with supra-molecular aggregates of water derived from Quantum Electro Dynamics (QED) and third model
- formation of nano-particles from the original solute plus solvent components. A careful scrutiny reveals that the second model, in effect, validates the first one through some special properties of water from QED and other considerations. Regarding nano-particles, they are bridges between bulk materials and atoms/molecules. Their number in a certain quantity of material taken for serial dilution will be less than what the Avogadro number suggests. Consequently, nano-particles of the starting material will cease to exist even before potency of 12c. This simple logic is sufficient to reject the third model though it escapes the notice of many pundits. Thus, we are left with only the first model - the Structural Model. Now, it remains to be established that structures can have medicinal value and cause cures.

\section{Answering the second question}

The chemical-free preparations of homoeopathy and nonhomoeopathic medicines with identifying chemical formula BOTH cure diseases. The curative agents may or may not have any distinct chemical formula. Logically, a substance is to be recognized as a medicine if it has the capability of curing disease(s). So, it needs a shift our focus from chemical formula and adopt a new approach for understanding medicines.

Different molecular structures are possible with same water having the chemical formula of $\mathrm{H}_{2} \mathrm{O}$, particularly with potentised homoeopathic medicines. We also note that due to various reasons the molecules of a living organism may change their form to some extent. But when this develops to a considerable extent it may be considered to be in a diseased state. With this understanding let us scrutinize action of water structures on bio-molecules in relation to how they can serve as medicines.

i. Here, another special property of water is of fundamental importance. All healthy bio-molecules fit in nicely within the hollows of water molecules in solid as well as liquid forms. Otherwise we could not preserve foods in refrigerators. But, the reaction of water towards the molecules whose form does not fit the structure of ice is quite different - it breaks the larger molecules and drives away the small ones. For this reason, the ice in Arctic Ocean is fresh-water ice- free from all salts. ${ }^{2}$ As such, it may safely be assumed that suitably structured water molecule of potentised medicines may bend near-matching bio-molecules to get a desired fit and may bring them to their healthy state.

ii. In fact, damaged bio-molecules are found to be repaired by bringing them to their healthy state with the help of tiny ice crystals. By freezing onto curved molecules the icicles straighten them out, giving them their usual configuration. ${ }^{4}$ This ref also tells about beneficial effects (like faster growth and higher immunity) on young birds and animals fed with melted-ice (called live water\} rich with ice crystals. [Boiled water is called dead water.]

iii. Structural fitting and template principle explain biological metabolic processes. This is quite well known to biologists. But, does it hold good for curative process as well? The answer is-Yes. For, we are not assuming anything new. We are only saying that water structures of medicines can also serve as correcting templates since they will be endowed with the special property mentioned above.

iv. Water of diseased human cells is found to be rather disordered as compared to water of healthy cells. ${ }^{47-51}$ It is an impression of diseased bio-molecules on cell water. Is the reverse also 
true - impression of suitably structured water on bio-molecule to revert it back to its healthy form? Here also the answer is -Yes. Introduction of suitable water structure into the body is very likely to bring the diseased bio-molecules to their normal condition and thereby restore health by virtue of the same special property of water.

It should be carefully noted here that any arbitrary water structure will not be a medicine. A radio set may be placed in a location full of all sorts of electromagnetic waves. But, the set will respond only to the frequency of the electromagnetic signal to which it is tuned. Similarly, structural influence (and hence, medical action) can occur only with closeness of structural matching/binding with biomolecules. Otherwise, it becomes a misfire.

So, the CLUE to medicinal value of high potency homoeomedicines seems to be 'structured water MOLECULES'. Cure follows from structural interaction of near-matching molecules. This concept embraces low potency homoeopathic medicines also as they contain chemicals with their well defined structures as well as structured water following potentisation process.

\section{Structures can serve as medicines}

This leads to the structural model of medicine: A substance is to be recognized as a medicine if it has the capability of curing disease(s) while its medicinal property is to be attributed to molecular structure of vehicle like water or of distinct chemical substance when it exists. It is to be noted here that all non-homoeopathic medicines (which are all un-potentised) and low potency $(<12 \mathrm{c})$ homoeopathic medicines have their own molecular structures (as they are not devoid of the starting material) for serving as medicines. In higher potencies $(>12 \mathrm{c})$ water structures are only available substances to serve as medicines. This forces us to appreciate the powerful role of structure in biological systems.

Further, let us look at some commoner facts supporting structural model.

a. If chemistry was the sole deciding factor, then we could not have Carbo-animalis and Graphites as two separate medicines in homoeopathy since the starting materials for both are chemically nothing but carbon. They differ in the structural arrangement of their constituent atoms.

b. The homoeopathic medicine called X-ray does not have any chemistry even in its lowest potency. Structural change of molecular arrangement of the vehicle stands out as the only possible explanation.

c. Homoeopathic medicines exposed to direct sunlight lose their medicinal value. Its only explanation left to us is melting/ disintegration of structures of the vehicle or the preservative at high temperature. It is likely to be similar to loss of structures of doped semiconductors getting melted at high temperature.

d. Homoeopathic medicines simply diluted in water (that is, without any preservative like ethyl alcohol) lose their medicinal value after 3-4days. This may be explained by spontaneous disintegration and reorganization of water structures at room temperature.

\section{Structural concept in non-homoeopathy}

The structural concept advanced above for homoeopathy has strong similarity to action of antibiotics through structural locking/ binding. Different antibiotics kill different bacteria by inhibiting their different functions such as protein synthesis, RNA synthesis, cell wall synthesis etc. ${ }^{52}$ Pathways, targets or action-sites vary from case to case. But the mechanism of action starts with structural matching/binding to specific bio-molecules. In some cases they have been identified. For example, penicillin (or other beta-lactams) bind to specific penicillin binding proteins (PBPs) in the cell wall and inhibit formation of cross links between peptidoglycan chains. This activates autolysins that degrade the cell wall, leading to bacterial cell death. Quinolones target DNA replication and repair by binding DNA gyrase complexed with DNA, which drives double-strand DNA break formation and cell death. ${ }^{53}$ Instead of destroying a cell from the outside in, like penicillin, some antibiotics block a cell's ability to make what it needs to proliferate from the inside out. Macrolide antibiotics are protein synthesis inhibitors; for example, the common macrolide antibiotic erythromycin works by binding to specific molecules- subunits- in a cell's ribosome, Destroying the cell's ability to form the proteins it needs for cell growth. Sulfa antibiotics (sulfonamides) have been used to battle bacterial infections since the 1930s. They target specific chemical reactions within a cell-the metabolic pathways -- by binding to an enzyme called dihydropteroate synthase (DHPS), which then blocks bacteria's ability to synthesize dihydriofolic acid. When this type of bacterial cell stops being able to metabolize folate, it can no longer grow or multiply. ${ }^{53}$ All of these start with binding with casespecific bio-molecules. The phenomenon of binding is the common feature in all these cases. Such bindings will obviously mean structural fitting and hence supporting the structural model of medicines.

\section{Conclusion}

That structures like bio-templates play important role in metabolic process is an established fact in biology. DNAs and RNAs are recognized as primary and secondary templates. But, when it comes to medicines, homoeopathic or non-homoeopathic, nobody thought of considering structures in a general way. People are mostly prone to think of medicines in terms of their chemical composition except in situations like action of antibiotics. But, every chemical formula has its corresponding physical structure. So, why can't we consider all medicinal actions as interaction between structures? The present author thinks that it is a better approach. It does not invalidate conventional chemistry but adds an extra dimension to it enabling us to interpret all medicinal actions successfully in terms of physical structure. High potency homoeo-medicines can be characterized only by molecular structures of their vehicle. Structures are obvious for Low potency homoeo-medicines and all non-homoeopathic medicines. Structures, whether of antibiotics or of other non-homoeopathic medicines or of low potency homoeopathic medicines or of water associated with high potency homoeopathic medicines, all can have medicinal effect. So, the structural model has a very strong case for generalized concept of medicines.

\section{Acknowledgements}

The infrastructural facilities of IIEST Shibpur; CEM Kolaghat; financial supports from UGC of Govt. of India; AYUSH of Govt. of India through CCRH and free supply of medicines etc. from HAPCO, Kolkata at different stages of this research are gratefully acknowledged.

\section{Conflict of interest}

The author declares no conflict of interest. 


\section{References}

1. Mahata CR. Scientific basis of potentised homoeopathic medicinestheoretical position and direction of future research. Indian Journal of Classical Homoeopathy. 2001:33-36.

2. Karapetyants M, Drakin S. Structure of Matter. Russia: Mir Publishers; 1974. p. 258-259.

3. Finkelnburg W. Structure of Matter. USA: Academic Press; 1964. p. $412-414$.

4. Sergeev B. Physiology for Everyone. Russia: Mir Publishers; 1973. p. $11-16$.

5. Singh PP, Chhabra HL. Topological investigation of the ethanol/water system and its implication for the mode of action of Homoeopathic Medicines. British Homoeopathic Journal. 1993;82(3):164-171.

6. Elia V, Germano R, Napoli E. Permanent Dissipative Structures in Water: The Matrix of Life? Experimental Evidences and their Quantum Origin. Curr Top Med Chem. 2015;15(6):559-571.

7. Lo SY, Geng X, Gann D. Evidence for the existence of stable waterclusters at room temperature and normal pressure. Phys Lett A. 2009;373(42):3872-3876.

8. Ho MW. Large Supramolecular Water Clusters Caught on Camera - A Review. WATER. 2014;6:1-12.

9. Marchettini N, Giudice E Del, Voeikov V, et al. Water: A medium where dissipative structures are produced by a coherent dynamics. J Theor Biol. 2010;265(4):511-516.

10. Giudice ED, Preparata G, Vitiello G. Water as a free electric dipole laser Phys Rev Lett. 1988;61:1085-1088.

11. Arani R, Bono I, Giudice ED, et al. QED Coherence and the thermodynamics of water. Int J Modern Phys. 1995;139:1813-1841.

12. Yinnon $\mathrm{C}$, Yinnon TT. Domains in aqueous solutions: Theory and experimental evidence. Modern Physics Letters B. 2009;23(16):1959 1973

13. Germano R. Water's quantum structures and life. Electromagnetic Biology and Medicine. 2015;34(2):133-137.

14. Bano I, Giudice ED, Gamberale L, et al. Emergence of the Coherent Structure of Liquid Water. Water. 2012;4:510-532.

15. http://www.i-sis.org.uk/water4.php

16. Elia V, Napoli E, Niccoli M. Physical-Chemical Study of Water in Contact with a hydrophilic polymer: Nafion. J Therm Anal Calorim. 2013;112(2):937-944.

17. Elia V, Ausanio G, Ninno AD, et al. Experimental evidence of stable aggregates of water at room temperature and normal pressure after iterative contact with Nafion polymer membrane. WATER. 2013;5:16-26.

18. Capolupo A, Giudice ED, Elia V, et al. Self-similarity properties of nafionized and filtered water and deformed coherent states. Int J Mod Phys B. 2013;28(3):14500076.

19. Elia V, Napoli E, Niccoli M. Calorimetric and Conductometric titrations of nanostructures of water molecules in Iteratively Filtered Water. JTAC. 2013; 111:815-821.

20. Cattaneo TMP, Vero S, Napoli E, et al. Influence of Filtration Process on Aqueous Nanostructures by NIR Spectroscopy. J Chem Eng. 2011;5:1046-1052.

21. Elia V, Ausanio G, Ninno AD, et al. Experimental Evidences of Stable Water Nanostructures At Standard Pressure And Temperature Obtained by Iterative Filtration. WATER. 2014;5:121-130.
22. Zheng JM, Chin WC, Khijniak E, et al. Surfaces and interfacial water: evidence that hydrophilic surfaces have long-range impact. Adv Colloid Interface Sci. 2006;127(1):19-27.

23. Giudice ED, Tedeschi A, Vitiello G, et al. Coherent structures in liquid water close to hydrophilic surfaces. J Phys ConfSer. 2013;442(1):012028.

24. Cacace CM, Elia L, Elia V, et al. Conductometric and pHmetric Titrations of Extremely Diluted Solutions Using $\mathrm{HCl}$ Solutions as Titrant:A Molecular Model. J Mol Liq. 2009;146(3):122-126.

25. Elia V, Napoli E, Niccoli M. A molecular model of interaction between extremely diluted solutions and $\mathrm{NaOH}$ solutions used as titrant:Conductometric and pHmetric titrations. J Mol Liq. 2010;148:4550

26. Elia V, Napoli E, Germano R. The 'Memory of water': an almost deciphered enigma - Dissipative structures in the extremely diluted aqueous solutions. Homeopathy. 2007;96(3):163-169.

27. Elia V, Napoli E. Dissipative Structures in Extremely Diluted Solutions of Homeopathic Medicines: A Molecular Model based on PhysicoChemical and Gravimetric evidences. Int J Des Nat. 2010;5:39-48.

28. Elia V, Ausanio G, Gentile F, et al. Experimental evidence of Stable Water Nanostructures in Extremely Diluted Solutions at Standard Pressure and Temperature. Homeopathy. 2014;103(1):44-50.

29. Yinnon TA, Elia V. Dynamics in perturbed very Dilute Aqueous Solutions: Theory and Experimental Evidence. Int J Modern Phys B. 2013;27(5):13500057.

30. Maity Tanmoy, Ghosh D, Mahata CR. Theory and Instrumentation related to Potentised Homoeopathic Medicines. IE(I) Journal- ID. 2007;88:27-31.

31. Maity T, Ghosh D, Mahata CR. Effect of Dielectric Dispersion on Potentised Homoeo-Medicines. Homeopathy. 2010;99(2):99-103.

32. Mahata CR. Dielectric Dispersion Studies Indicate Change in Structure of Water by Potentised Homeopathic Medicines. IE(I). 2013;93(4):23135 .

33. Mahata CR. Dielectric Dispersion Studies of Some Potentised Homoeopathic Medicines Reveal Structured Vehicle. Homeopathy. 2013;102(4):262-267.

34. Roy Rustum, Tiller WA, Bell Iris, et al. The structure of liquid water; novel insights from materials research; potential relevance to homeopathy. Materials Research Innovations Online. 2005:577-608.

35. Rao Manju Lata, Roy Rustum, Bell Iris R, et al. The defining role of structure (including epitaxy) in the plausibility of homeopathy. Homeopath. 2007;96(3):175-182.

36. Rey L. Thermo-luminescence of ultra-high dilutions of lithium chloride and sodium chloride. Physica. 2003;323:67-74.

37. Elia V, Ausanio G, Gentile F, et al. Experimental evidence of stable water nanostructures in extremely dilute solutions, at standard pressure and temperature. Homeopath. 2014;103(1):44-50.

38. Montagnier L, Giudice ED, Jamal A“1ssa, et al. Transduction of DNA information through water and electromagnetic waves. Electromagnetic Biology and Medicine. 2014:1-11.

39. Barnard GO. Microdose paradox -a new concept. J American Inst Homeopath. 1965;58(7):205-212.

40. Thomas Horder's. Communication to the Royal Society of Medicine published as "The electronic reactions of Abrams". The $\mathrm{Br}$ Med J. 1925;1(3343):179-185

41. Chattopadhyay R, Sadhukhan M, Pal A, et al. On electromagnetic signals from vastly diluted DNA solutions, potentised medicines and even metals. Wesleyan Journal of Research. 2012;5(1):50-55. 
42. Anagnostatos GS, Vithoulkas G, Garzonis P, et al. A working hypothesis for homoeopathic microdiluted remedies. Berlin J Resear Homoeopathy. 1991;1(3):141-147.

43. http://www1.1sbu.ac.uk/water/water structure science.html

44. Davenas E, Beauvais F, Amara J, et al. Human basophil degranulation triggered by very dilute antiserum against IgE. Nature. 1988;333(6176):816-818.

45. Mahata CR. Structural model explains high potency homoeopathic medicines and leads to a generalized understanding of medicines. Intl $J$ CAM. 2015;1(2):1-3.

46. Bellavite P, Marzotto M, Olioso D, et al. High-dilution effects revisited. 1. Physicochemical aspects. Homeopathy. 2014;103(1):4-21.

47. Chaughule R S. NMR:A New Eye on Cancer. Science Today. 1978:2327.
48. Damadian R. Tumor Detection by NMR. Science. 1971;171:1151-1153.

49. Hollis DP, Economou JS, Parks LC, et al. Nuclear Magnetic Resonance Studies of several experimental and human malignant tumors. Cancer Res. 1973;33(9):2156-2160.

50. Chaughule RS, Kasturi SR, Vijayaraghavan R, et al. Normal and Malignant Tissues: An Investigation by Pulsed NMR. Indian J Biochem Biophy. 1974; 11:256.

51. Ranade SS, Shah S, Korgaonkar KS, et al. Absence of Correlation between Spin-Lattice Relaxation Times and water content in human tumor tissues. Physiol Chem Phys. 1976;8(2):131-134.

52. Kohanski MA, Dwyer DJ, Hayete B, et al. A Common Mechanism of Cellular Death Induced by Bactericidal Antibiotics. Cell. 2007;130(5):797-810.

53. Maria T. How do antibiotics work? 2015. 